\title{
Impact of the Doppler Effect on the Capacity of Massive MIMO Uplink Systems: OFDM Versus FBMC/OQAM
}

\author{
Alexis Bazin \\ Orange Labs \\ Cesson-Sévigné, France \\ Email: alexis.bazin@orange.com
}

\author{
Bruno Jahan \\ Orange Labs \\ Cesson-Sévigné, France \\ Email: bruno.jahan@orange.com
}

\author{
Maryline Hélard \\ IETR, INSA \\ Rennes, France \\ Email: maryline.helard@insa-rennes.fr
}

\begin{abstract}
This paper studies the impact of the Doppler effect on the capacity of an uplink communication between one or several user(s) with mobility and a base station. The OFDM and the FBMC/OQAM modulations are investigated. Moreover, massive MIMO techniques are used at the receiver side to increase the SINR and to allow for simultaneous multiuser communications. An analytic study is carried out to highlight the impact of the Doppler effect on the achievable rate of this system and to bring out benefits and drawbacks of both modulations. On the one hand, the OFDM modulation presents a loss of spectral efficiency because of its cyclic prefix and a limited robustness against the Doppler effect. On the other hand, the FBMC/OQAM performance is restricted by its intrinsic interference due to the frequency selectivity of the channel. These two modulations are compared under different mobility scenarios and the ability of the FBMC/OQAM modulation to significantly increase the achievable rate per user compared to the OFDM modulation is highlighted, especially for high speed scenarios.
\end{abstract}

\section{INTRODUCTION}

In [1], the NGMN Alliance anticipated the future needs regarding wireless networks, which should be taken into account for the development of the fifth generation of cellular networks $(5 \mathrm{G})$. In particular, users with high mobility such as cars and trains are expected to benefit from better quality of service in the coming years. In [2], the Radiocommunication Sector of International Telecommunication Union (ITU-R) recommends to be able to communicate with a user up to 500 $\mathrm{km} / \mathrm{h}$ under vehicle-to-vehicle $(\mathrm{V} 2 \mathrm{~V}$ ) or vehicle-to-everything (V2X) scenarios. Therefore, new techniques and waveforms are investigated in order to overcome these constraints.

The filter-bank multicarrier (FBMC) modulation and in particular the FBMC with offset quadrature amplitude modulation (OQAM) [3], [4] is an interesting candidate thanks to its good spectral and temporal localizations. Indeed, the FBMC/OQAM waveform does not require strict synchronization [5], presents a good robustness against the Doppler effect as discussed in [6]-[8] and thus is particularly suitable for high mobility applications. Furthermore, compared to the orthogonal frequency division multiplexing (OFDM) modulation, the FBMC/OQAM waveform does not need any cyclic prefix (CP) and thus achieves a better spectral efficiency.
In addition, massive MIMO techniques, which use a large number of antennas at the base station side, have gained great interest in the past years [9]. They can be used for uplink multiuser communication to manage multiuser interference and to improve the spectral and energy efficiency [10]. Indeed, as stated in [11] and further improved in [12], [13], with an unlimited number of antennas, noise and multiuser effects vanished and the only remaining interference is inter-cellular interference (pilot contamination).

Only few studies combine the FBMC waveform and massive MIMO for uplink. In [14], [15] the authors associate multiuser massive MIMO methods for uplink and two FBMC techniques, cosine modulated multitone (CMT) and frequency spreading FBMC (FS-FBMC). They highlight the capacity of massive MIMO to reduce the frequency selectivity of the channel and called it "self equalization". According to this results, one may wonder if massive MIMO has also an effect on the time selectivity and therefore on the Doppler effect. Nevertheless, to the best of the authors' knowledge, studies on performance of massive MIMO systems rarely take into account the Doppler effect. Thus, as the FBMC/OQAM modulation is well suited for mobility applications, studying its performance is of great interest.

In this paper we compare the FBMC/OQAM and the OFDM modulations for massive MIMO uplink systems and for users with mobility in a $5 \mathrm{G}$ context. The system model is presented in part II. In part III, a theoretical analysis is conducted to compare these two modulations in terms of signal to interference-plus-noise ratio (SINR) and achievable rate per user. Then, in part IV, numerical results allow for conclusion concerning the best modulation as a function of the number of receive antennas and the user mobility.

The following notations are used. The symbol ? indicates variables for the FBMC/OQAM modulation. Vectors and matrices are represented in boldface letters. The Euclidean norm of a matrix or vector is denoted by $\|\|,.(.)^{T}$ indicates their transpose and $(.)^{H}$ stands for their conjugate transpose. The identity matrix of size $n \times n$ is defined by $\mathbf{I}_{n}$. Finally, the conjugate value of a complex number is denoted by $(.)^{*}$, its expected value by $E[$.$] , its real part by \Re[$.$] and its imaginary$ 


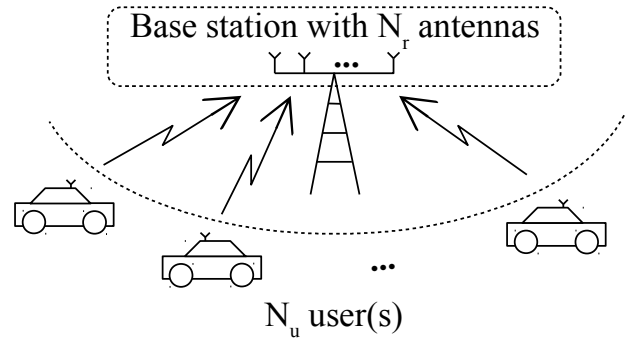

Fig. 1. Scenario considered for this paper: an uplink communication between $N_{u}$ users with mobility and a base station equipped with $N_{r}$ antennas.

part by $\Im[$.$] .$

\section{SYSTEM MODEL}

For this study, an uplink scenario is considered with simultaneous transmissions between $N_{u}$ users and a base station equipped with $N_{r}$ receive antennas. As in [14], [15] and for the sake of simplicity, we assume that all users have a single antenna, are moving at the same speed and are at the same distance from the base station. The scenario is described by Figure 1. The receive antennas are sufficiently spaced so that all propagation channels between a user and a receive antenna are considered to be independent from each other. Moreover, a perfect channel estimation at the receiver side is assumed. At the base station, a MMSE processing is carried out in order to equalize the channel and to separate users' data. Indeed, the MMSE processing performs the best performance according to [14]. For the rest of this paper, we set $M$ as the number of subcarriers and $F_{0}$ as the intercarrier spacing. The symbol duration is therefore $T_{0}=1 / F_{0}$ and the sampling time is $T_{s}=T_{0} / M$. The index for the time-domain signals and propagation channel paths is $k$. The propagation channel model has $L_{h}$ paths and the value along time of the $l^{\text {th }}$ path between the user $n_{u}$ and the antenna $n_{r}$ is denoted by $h_{l}^{n_{u}, n_{r}}[k]$. In part II-A, we define the OFDM link and in part II-B the FBMC/OQAM link.

\section{A. OFDM link}

The system model for an OFDM link is depicted in Figure 2. After the modulation process, the time domain baseband signal of user $n_{u}$ is:

$$
s^{n_{u}}[k]=\frac{1}{\sqrt{M}} \sum_{m=0}^{M-1} c^{n_{u}}[m] e^{j 2 \pi \frac{m}{M} k},
$$

with $c^{n_{u}}[m]$ the complex data on subcarrier $m$ of variance $\sigma_{c}^{2}$. A CP is added to counteract the propagation channel delay spread. The received signal on the antenna $n_{r}$ is defined by:

$$
r^{n_{r}}[k]=\left(\sum_{n_{u}=0}^{N_{u}-1} \sum_{l=0}^{L_{h}-1} s^{n_{u}}[k-l] h_{l}^{n_{u}, n_{r}}[k]\right)+\eta^{n_{r}}[k],
$$

with $\eta^{n_{r}}[k]$ the noise component on this antenna. Then, a demodulation process is performed on each receive antenna. For a given subcarrier $m_{0}$, we set $c_{p}^{n_{u}}=c^{n_{u}}\left[m_{0}+p\right]$ the data

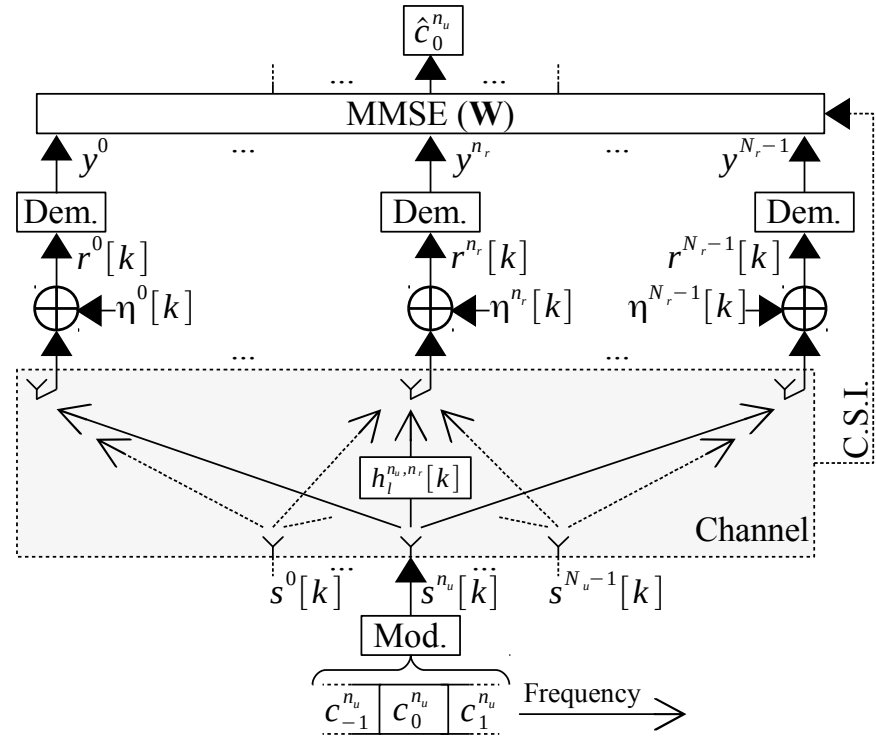

Fig. 2. System model for an uplink communication with an OFDM modulation, $N_{u}$ users and $N_{r}$ antennas at the base station side.

transmitted on subcarrier $\left(m_{0}+p\right)$. The received data on the antenna $n_{r}$ is:

$$
\begin{aligned}
y^{n_{r}} & =\frac{1}{\sqrt{M}} \sum_{k=0}^{M-1} r^{n_{r}}[k] e^{-j 2 \pi \frac{m_{0}}{M} k} \\
& =\left(\sum_{n_{u}=0}^{N_{u}-1} \sum_{p} H_{p}^{n_{u}, n_{r}} c_{p}^{n_{u}}\right)+b^{n_{r}},
\end{aligned}
$$

with $b^{n_{r}}$ the frequency domain noise component of variance $\sigma_{b}^{2}$ and with:

$$
H_{p}^{n_{u}, n_{r}}=\frac{1}{M} \sum_{k=0}^{M-1} \sum_{l=0}^{L_{h}-1} h_{l}^{n_{u}, n_{r}}[k] e^{j 2 \pi \frac{p}{M} k} e^{-j 2 \pi \frac{m_{0}+p}{M} l} .
$$

For the link between the user $n_{u}$ and the antenna $n_{r}, H_{0}^{n_{u}, n_{r}}$ represents the useful component of the channel when receiving the data $c_{0}^{n_{u}}$ on subcarrier $m_{0}$ and $H_{p \neq 0}^{n_{u}, n_{r}}$ stand for the components of the channel that lead to intercarrier interference on $c_{0}^{n_{u}}$ coming from $c_{p \neq 0}^{n_{u}}$. Let us define the channel matrices:

$$
\left\{\begin{array}{l}
\mathbf{H}_{p}^{n_{u}}=\left[\begin{array}{lllll}
H_{p}^{n_{u}, 0} & H_{p}^{n_{u}, 1} & \ldots & H_{p}^{n_{u}, N_{r}-1}
\end{array}\right]^{T}, \\
\mathbf{H}_{p}=\left[\begin{array}{llll}
\mathbf{H}_{p}^{0} & \mathbf{H}_{p}^{1} & \cdots & \mathbf{H}_{p}^{N_{u}-1}
\end{array}\right] .
\end{array}\right.
$$

The received data vector is $\mathbf{y}=\left[\begin{array}{llll}y^{0} & y^{1} & \cdots & y^{N_{r}-1}\end{array}\right]^{T}$ and the noise vector is $\mathbf{b}=\left[\begin{array}{llll}b^{0} & b^{1} & \cdots & b^{N_{r}-1}\end{array}\right]^{T}$. The MMSE decoding matrix is defined by:

$$
\begin{aligned}
\mathbf{W} & =\mathbf{H}_{0}\left(\mathbf{H}_{0}^{H} \mathbf{H}_{0}+\rho^{-1} \mathbf{I}_{N_{u}}\right)^{-1} \\
& =\left[\begin{array}{llll}
\mathbf{W}^{0} & \mathbf{W}^{1} & \cdots & \mathbf{W}^{N_{u}-1}
\end{array}\right],
\end{aligned}
$$

with $\rho=\sigma_{c}^{2} / \sigma_{b}^{2}$, the signal to noise ratio (SNR) which is the same for all users. The estimated received data after MMSE processing $\hat{\mathbf{c}}_{0}$ are defined by:

$$
\hat{\mathbf{c}}_{0}=\mathbf{W}^{H} \mathbf{y}=\left[\begin{array}{llll}
\hat{c}_{0}^{0} & \hat{c}_{0}^{1} & \cdots & \hat{c}_{0}^{N_{u}-1}
\end{array}\right]^{T} .
$$

In a multiuser scenario, a given user $n_{u}$ will experience interference coming from the $\left(N_{u}-1\right)$ other users. The indexes 


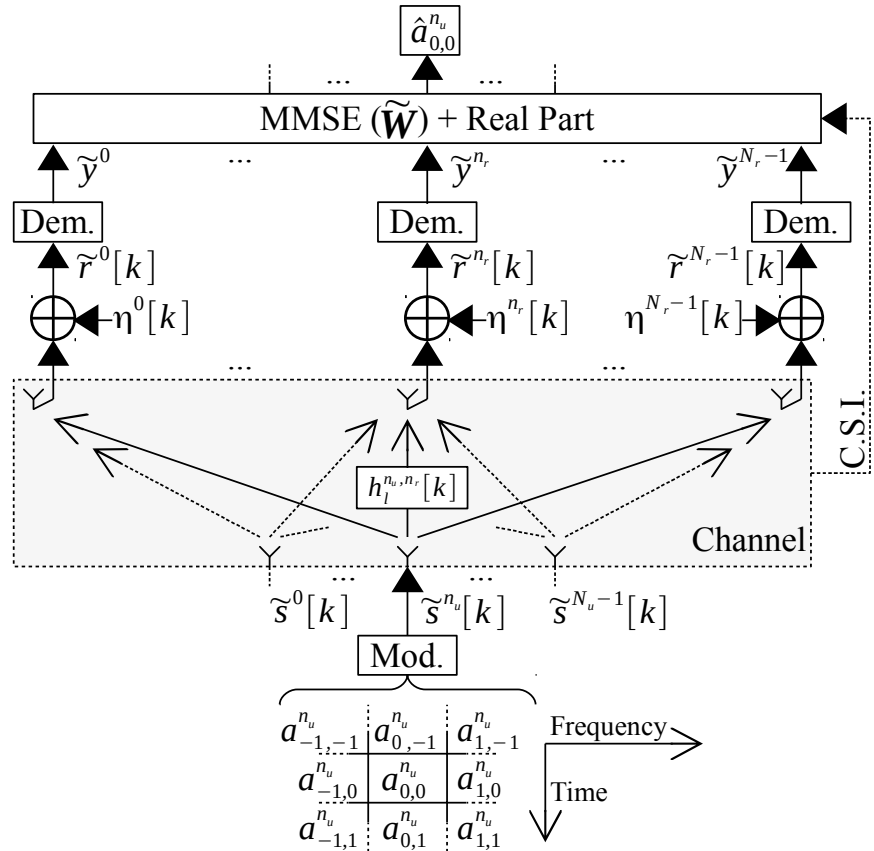

Fig. 3. System model for an uplink communication with an FBMC/OQAM modulation, $N_{u}$ users and $N_{r}$ antennas at the base station side.

of this interfering users are defined by $n_{u}^{\prime} \neq n_{u}$. Thus, for the user $n_{u}$, the received estimated data is therefore:

$$
\begin{aligned}
\hat{c}_{0}^{n_{u}} & =\left(\mathbf{W}^{n_{u}}\right)^{H} \mathbf{H}_{0}^{n_{u}} c_{0}^{n_{u}} \\
& +\sum_{p \neq 0}\left(\mathbf{W}^{n_{u}}\right)^{H} \mathbf{H}_{p}^{n_{u}} c_{p}^{n_{u}} \\
& +\sum_{n_{u}^{\prime} \neq n_{u}} \sum_{p}\left(\mathbf{W}^{n_{u}}\right)^{H} \mathbf{H}_{p}^{n_{u}^{\prime}} c_{p}^{n_{u}^{\prime}} \\
& +\left(\mathbf{W}^{n_{u}}\right)^{H} \mathbf{b},
\end{aligned}
$$

where the first, second, third and last lines of this equation respectively stand for the useful part, the Doppler interference part, the multiuser interference part and the noise part of the signal.

\section{B. FBMC/OQAM link}

In an FBMC/OQAM system, real data are sent every $T_{0} / 2$ seconds. Therefore, compared to the OFDM modulation, the orthogonality condition is only valid for the real field. Thus the symbols can be shaped by a large set of prototype filters with an increase of the complexity in return [8]. We define the discrete time formulation of this filter by $g[k]$ and its length by $L_{g}$. Figure 3 depicts the system model for an FBMC/OQAM modulation. The baseband transmitted discrete-time FBMC/OQAM signal for the user $n_{u}$ can be expressed as in [4] by $\tilde{s}^{n_{u}}[k]$ :

$$
\tilde{s}^{n_{u}}[k]=\sum_{n \in \mathbb{Z}} \sum_{m=0}^{M-1} a^{n_{u}}[m, n] g_{m, n}[k],
$$

with $a^{n_{u}}[m, n]$ the real data of variance $\sigma_{a}^{2}=\sigma_{c}^{2} / 2$ sent at the frequency and time indexes $m$ and $n$ respectively and $g_{m, n}[k]$ defined by:

$$
g_{m, n}[k]=g[k-n M / 2] e^{j 2 \pi \frac{m}{M}\left(k-\frac{L_{g}-1}{2}\right)} e^{j \phi[m, n]} .
$$

In (10) the phase term $\phi[m, n]$ is defined by:

$$
\phi[m, n]=(m+n) \pi / 2+k_{\phi}[m, n] \pi, k_{\phi}[m, n] \in \mathbb{Z} .
$$

Similarly to (2), the received signal on the antenna $n_{r}$ is:

$$
\tilde{r}^{n_{r}}[k]=\left(\sum_{n_{u}=0}^{N_{u}-1} \sum_{l=0}^{L_{h}-1} \tilde{s}^{n_{u}}[k-l] h_{l}^{n_{u}, n_{r}}[k]\right)+\eta^{n_{r}}[k] .
$$

For the frequency and time indexes $\left(m_{0}, n_{0}\right)$, we define $a_{p, q}^{n_{u}}=$ $a^{n_{u}}\left[m_{0}+p, n_{0}+q\right]$ and the received data on the antenna $n_{r}$ after demodulation is:

$$
\begin{aligned}
\tilde{y}^{n_{r}} & =\sum_{k=0}^{M-1} \tilde{r}^{n_{r}}[k] g_{m_{0}, n_{0}}^{*}[k] \\
& =\left(\sum_{n_{u}=0}^{N_{u}-1} \sum_{p, q} \tilde{H}_{p, q}^{n_{u}, n_{r}} a_{p, q}^{n_{u}}\right)+b^{n_{r}}
\end{aligned}
$$

with:

$\left\{\begin{array}{l}\tilde{H}_{p, q}^{n_{u}, n_{r}}=\sum_{l=0}^{L_{h}-1} \tilde{h}_{p, q}^{n_{u}, n_{r}}[l] e^{-j 2 \pi \frac{m_{0}+p}{M} l} e^{-j \pi \frac{p}{M}\left(L_{g}-1\right)} e^{j \Psi_{p, q}}, \\ \tilde{h}_{p, q}^{n_{u}, n_{r}}[l]=\sum_{k=0}^{L_{g}-1} g[k-l-q M / 2] g[k] h_{l}^{n_{u}, n_{r}}[k] e^{j 2 \pi \frac{p}{M} k} \\ \Psi_{p, q}=\phi\left[m_{0}+p, n_{0}+q\right]-\phi\left[m_{0}, n_{0}\right]+\pi p n_{0} .\end{array}\right.$

Compared to (4), $\tilde{H}_{(p, q) \neq(0,0)}^{n_{u}, n_{r}}$ represent the channel components that lead to intercarrier and intersymbol interference. Indeed, as it does not use any CP, an FBMC/OQAM modulation also suffers from intrinsic interference caused by the frequency selectivity of the channel. We now define the channel matrices:

$$
\left\{\begin{aligned}
\tilde{\mathbf{H}}_{p, q}^{n_{u}} & =\left[\begin{array}{llll}
\tilde{H}_{p, q}^{n_{u}, 0} & \tilde{H}_{p, q}^{n_{u}, 1} & \cdots & \tilde{H}_{p, q}^{n_{u}, N_{r}-1}
\end{array}\right]^{T}, \\
\tilde{\mathbf{H}}_{p, q} & =\left[\begin{array}{llll}
\tilde{\mathbf{H}}_{p, q}^{0} & \tilde{\mathbf{H}}_{p, q}^{1} & \cdots & \tilde{\mathbf{H}}_{p, q}^{N_{u}-1}
\end{array}\right],
\end{aligned}\right.
$$

and the received data vector $\tilde{\mathbf{y}}=\left[\begin{array}{llll}\tilde{y}^{0} & \tilde{y}^{1} & \ldots & \tilde{y}^{N_{r}-1}\end{array}\right]^{T}$. The MMSE decoding matrix is defined by:

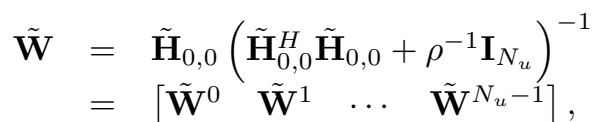

so that:

$$
\hat{\mathbf{a}}_{0,0}=\Re\left[\tilde{\mathbf{W}}^{H} \tilde{\mathbf{y}}\right]=\left[\begin{array}{llll}
\hat{a}_{0,0}^{0} & \hat{a}_{0,0}^{1} & \cdots & \hat{a}_{0,0}^{N_{u}-1}
\end{array}\right]^{T} .
$$

As only the real part is retained, the noise power is reduced to $\sigma_{b}^{2} / 2$ and the value of the SNR is the same as in an OFDM link, meaning $\rho=\sigma_{a}^{2} /\left(\sigma_{b}^{2} / 2\right)=\sigma_{c}^{2} / \sigma_{b}^{2}$. For a given user $n_{u}$, the received estimated data is therefore:

$$
\begin{aligned}
\hat{a}_{0,0}^{n_{u}} & =\Re\left[\left(\tilde{\mathbf{W}}^{n_{u}}\right)^{H} \tilde{\mathbf{H}}_{0,0}^{n_{u}}\right] a_{0,0}^{n_{u}} \\
& +\sum_{(p, q) \neq(0,0)} \Re\left[\left(\tilde{\mathbf{W}}^{n_{u}}\right)^{H} \tilde{\mathbf{H}}_{p, q}^{n_{u}}\right] a_{p, q}^{n_{u}} \\
& +\sum_{n_{u}^{\prime} \neq n_{u}} \sum_{(p, q)} \Re\left[\left(\tilde{\mathbf{W}}^{n_{u}}\right)^{H} \tilde{\mathbf{H}}_{p, q}^{n_{u}^{\prime}}\right] a_{p, q}^{n_{u}^{\prime}} \\
& +\Re\left[\left(\tilde{\mathbf{W}}^{n_{u}}\right)^{H} \mathbf{b}\right] .
\end{aligned}
$$

As for (8), the first, second, third and fourth lines of (18) respectively stand for the useful part, the intrinsic and Doppler effect interference part, the multiuser interference part and the noise part of the receive signal. 
The impact of the Doppler effect can now be analyzed through a theoretical analysis of the OFDM and FBMC/OQAM performance in the studied context.

\section{THEORETICAL PERFORMANCE ANALYSIS}

The aim of this part is to highlight the impact of the mobility as well as the benefits and limitations of the OFDM and FBMC/OQAM modulations. Therefore, based on their SINR, we compare the achievable rate per user for both modulations, for a finite and an infinite number of antennas at the base station.

\section{A. Signal to interference-plus-noise ratio}

For the OFDM modulation, the SINR $\gamma^{n_{u}}$ is computed for the user $n_{u}$ by using (8) and for the FBMC/OQAM modulation, the SINR $\tilde{\gamma}^{n_{u}}$ is obtained by using (18):

$$
\left\{\begin{array}{l}
\gamma^{n_{u}}=P_{U}^{n_{u}} /\left(P_{I}^{n_{u}}+P_{M U}^{n_{u}}+P_{N}^{n_{u}}\right), \\
\tilde{\gamma}^{n_{u}}=\tilde{P}_{U}^{n_{u}} /\left(\tilde{P}_{I}^{n_{u}}+\tilde{P}_{M U}^{n_{u}}+\tilde{P}_{N}^{n_{u}}\right),
\end{array}\right.
$$

with:

$$
\left\{\begin{aligned}
P_{U}^{n_{u}} & =\left|\left(\mathbf{W}^{n_{u}}\right)^{H} \mathbf{H}_{0}^{n_{u}}\right|^{2} \sigma_{c}^{2}, \\
\tilde{P}_{U}^{n_{u}} & =\Re\left[\left(\tilde{\mathbf{W}}^{n_{u}}\right)^{H} \tilde{\mathbf{H}}_{0,0}^{n_{u}}\right]^{2} \sigma_{a}^{2},
\end{aligned}\right.
$$

defining the useful power,

$$
\left\{\begin{aligned}
P_{M U}^{n_{u}} & =\sum_{n_{u}^{\prime} \neq n_{u}} \sum_{p}\left|\left(\mathbf{W}^{n_{u}}\right)^{H} \mathbf{H}_{p}^{n_{u}^{\prime}}\right|^{2} \sigma_{c}^{2}, \\
\tilde{P}_{M U}^{n_{u}} & =\sum_{n_{u}^{\prime} \neq n_{u}} \sum_{p, q} \Re\left[\left(\tilde{\mathbf{W}}^{n_{u}}\right)^{H} \tilde{\mathbf{H}}_{p, q}^{n_{u}^{\prime}}\right]^{2} \sigma_{a}^{2},
\end{aligned}\right.
$$

the multiuser interference power and

$$
\left\{\begin{aligned}
P_{N}^{n_{u}} & =\left\|\mathbf{W}^{n_{u}}\right\|^{2} \sigma_{b}^{2}, \\
\tilde{P}_{N}^{n_{u}} & =\left\|\tilde{\mathbf{W}}^{n_{u}}\right\|^{2} \sigma_{b}^{2} / 2,
\end{aligned}\right.
$$

the noise power. The intrinsic interference and the Doppler effect interference powers are defined by:

$$
\left\{\begin{aligned}
P_{I}^{n_{u}} & =\sum_{p \neq 0}\left|\left(\mathbf{W}^{n_{u}}\right)^{H} \mathbf{H}_{p}^{n_{u}}\right|^{2} \sigma_{c}^{2}, \\
\tilde{P}_{I}^{n_{u}} & =\sum_{(p, q) \neq(0,0)} \Re\left[\left(\tilde{\mathbf{W}}^{n_{u}}\right)^{H} \tilde{\mathbf{H}}_{p, q}^{n_{u}}\right]^{2} \sigma_{a}^{2},
\end{aligned}\right.
$$

and are induced by the time and frequency selectivity of the channel. Since for the OFDM modulation the SINR does not take into account the loss of spectral efficiency, the achievable rate per user is a more fair metric and is computed in the next subsection.

\section{B. Achievable rate per user}

For the OFDM (resp. FBMC/OQAM) modulation, the achievable rate per user $R$ (resp. $\tilde{R})$ is defined by:

$$
\left\{\begin{array}{l}
R=\alpha \times E\left[\log _{2}\left(1+\gamma^{n_{u}}\right)\right], \alpha<1 \\
\tilde{R}=E\left[\log _{2}\left(1+\tilde{\gamma}^{n_{u}}\right)\right],
\end{array}\right.
$$

with $\alpha=T_{0} /\left(T_{0}+T_{C P}\right), T_{C P}$ being the length of the CP. For a low SNR, a moderate speed and if $N_{r}$ is low enough, the intrinsic interference and the Doppler effect interference can be neglected compared to the noise and multiuser interference and we have:

$$
\left\{\begin{array}{l}
\left(P_{M U}^{n_{u}}+P_{N}^{n_{u}}\right) \gg P_{I}^{n_{u}}, \\
\left(\tilde{P}_{M U}^{n_{u}}+\tilde{P}_{N}^{n_{u}}\right) \gg \tilde{P}_{I}^{n_{u}} .
\end{array}\right.
$$

Under this condition, one can see that the problem is simplified and is comparable to the one described in [14] where the authors showed that the OFDM and FBMC waveforms lead to the same SINR $\left(\gamma^{n_{u}}=\tilde{\gamma}^{n_{u}}\right)$. Furthermore, according to [11], the SINR for the OFDM modulation increases as the value of $N_{r}$ increases. Therefore, we have $R=\alpha \times \tilde{R}$ and the higher the value of $N_{r}$, the higher the value of $R$ and thus the higher the difference $(\tilde{R}-R)$. Thereby, the FBMC/OQAM modulation should outperform the OFDM modulation, thanks to the lack of CP.

However, above a certain value for $N_{r}$, (25) are not valid anymore and the performance of the OFDM and FBMC/OQAM modulations are dependent on $P_{I}^{n_{u}}$ and $\tilde{P}_{I}^{n_{u}}$ and thus on the time and frequency selectivity of the channel. Moreover, for a high SNR or under high speed conditions, (25) are never valid even with a moderate number of antennas at the base station. Therefore, in a massive MIMO system with a large number of antennas at the base station and in mobility conditions, the asymptotic achievable rate per user (when $N_{r} \rightarrow+\infty$ ) could reveal to be an interesting metric as developed in the next section.

\section{Asymptotic values}

When $N_{r}$ tends towards infinity, multiuser interference (when $n_{u}^{\prime} \neq n_{u}$ ) vanishes as stated in [11] and owing to the law of large numbers, we have $\forall n_{u}, n_{r}$ :

$$
\left\{\begin{array}{l}
\lim _{N_{r} \rightarrow \infty}\left\{\left(\mathbf{H}_{0}^{n_{u}^{\prime}}\right)^{H} \mathbf{H}_{0}^{n_{u}}\right\}=0, \\
\lim _{N_{r} \rightarrow \infty}\left\{\left(\tilde{\mathbf{H}}_{0,0}^{n_{u}^{\prime}}\right)^{H} \tilde{\mathbf{H}}_{0,0}^{n_{u}}\right\}=0, \\
\lim _{N_{r} \rightarrow \infty}\left\{\left\|\mathbf{H}_{0}^{n_{u}}\right\|^{2}\right\}=N_{r} E\left[\left|H_{0}^{n_{u}, n_{r}}\right|^{2}\right], \\
\lim _{N_{r} \rightarrow \infty}\left\{\left\|\tilde{\mathbf{H}}_{0,0}^{n_{u}}\right\|^{2}\right\}=N_{r} E\left[\left|\tilde{H}_{0,0}^{n_{u}, n_{r}}\right|^{2}\right] .
\end{array}\right.
$$

Thus $\left(\mathbf{H}_{0}^{H} \mathbf{H}_{0}+\rho^{-1} \mathbf{I}_{N_{u}}\right)$ and $\left(\tilde{\mathbf{H}}_{0,0}^{H} \tilde{\mathbf{H}}_{0,0}+\rho^{-1} \mathbf{I}_{N_{u}}\right)$ become diagonal and we have:

$$
\left\{\begin{array}{l}
\lim _{N_{r} \rightarrow \infty}\left\{\mathbf{W}^{n_{u}}\right\}=\mathbf{H}_{0}^{n_{u}}\left(N_{r} E\left[\left|H_{0}^{n_{u}, n_{r}}\right|^{2}\right]+\rho^{-1}\right)^{-1}, \\
\lim _{N_{r} \rightarrow \infty}\left\{\tilde{\mathbf{W}}^{n_{u}}\right\}=\tilde{\mathbf{H}}_{0,0}^{n_{u}}\left(N_{r} E\left[\left|\tilde{H}_{0,0}^{n_{u}, n_{r}}\right|^{2}\right]+\rho^{-1}\right)^{-1} .
\end{array}\right.
$$

Moreover, as the multiuser and noise components become negligible when $N_{r}$ tends towards infinity [11], the asymptotic achievable rates per user $R_{\infty}$ and $\tilde{R}_{\infty}$, for the OFDM and FBMC/OQAM modulations respectively, are defined by:

$$
\left\{\begin{array}{l}
R_{\infty}=\alpha \times \log _{2}\left(1+\frac{\left(\mu_{0}^{\Re}\right)^{2}}{\sum_{p \neq 0}\left(\mu_{p}^{\Re}\right)^{2}+\left(\mu_{p}^{\Im}\right)^{2}}\right), \\
\tilde{R}_{\infty}=\log _{2}\left(1+\frac{\left(\tilde{\mu}_{0,0}^{\Re}\right)^{2}}{\sum_{(p, q) \neq(0,0)}\left(\tilde{\mu}_{p, q}^{\Re}\right)^{2}}\right),
\end{array}\right.
$$


with $\forall n_{u}, n_{r}$ :

$$
\left\{\begin{aligned}
\left(\mu_{p}^{\Re}\right)^{2} & =E\left[\Re\left[\left(H_{0}^{n_{u}, n_{r}}\right)^{*} H_{p}^{n_{u}, n_{r}}\right]\right]^{2}, \\
\left(\mu_{p}^{\Im}\right)^{2} & =E\left[\Im\left[\left(H_{0}^{n_{u}, n_{r}}\right)^{*} H_{p}^{n_{u}, n_{r}}\right]\right]^{2}, \\
\left(\tilde{\mu}_{p, q}^{\Re}\right)^{2} & =E\left[\Re\left[\left(\tilde{H}_{0,0}^{n_{u}, n_{r}}\right)^{*} \tilde{H}_{p, q}^{n_{u}, n_{r}}\right]\right]^{2} .
\end{aligned}\right.
$$

$\tilde{R}_{\infty}$ is impacted by the intrinsic interference and by the Doppler effect, while $R_{\infty}$ is only limited by the Doppler effect. However, as the FBMC/OQAM waveform is less sensitive to the Doppler effect (see [6]-[8]), in high mobility conditions we might have $\tilde{R}_{\infty} \geq R_{\infty}$.

To conclude, the FBMC/OQAM modulation can benefit from its better spectral efficiency. However, the Doppler effect has an impact as the performances of both the OFDM and FBMC/OQAM modulations are limited by the time selectivity of the channel. Moreover, the FBMC/OQAM waveform is also impacted by the frequency selectivity of the channel because of the lack of CP. Therefore the choice between the OFDM and FBMC/OQAM modulations is not straightforward in an analytic point of view as there is a trade-off between the time and frequency selectivity of the channel. Thus, simulations are necessary to accurately compare those two waveforms.

\section{Simulation RESUlts}

In this part, we numerically compare the achievable rate per user for the OFDM and the FBMC/OQAM modulations as a function of the number of users $N_{u}$, the number of receive antennas at the base station side $N_{r}$ and the speed of the users. The FBMC/OQAM modulation uses the TFL1 (Time Frequency Localization) filter of length $T_{0}$ defined in [16], which is designed to combine a small length and a good time-frequency localization in order to counteract the time selectivity of the channel. Classical values for LTE communications are chosen, a FFT size of $M=1024$ with 600 active subcarriers, a subcarrier spacing of $F_{0}=15 \mathrm{KHz}$ and a CP length of $T_{C P}=4.7 \mu s$ for the OFDM modulation. Moreover, the central frequency is set to $6 \mathrm{GHz}$ and the SNR to $\rho=10 \mathrm{~dB}$.

\section{A. Connected car scenario}

We first set the speed of the users to $50 \mathrm{~km} / \mathrm{h}$ (urban configuration) and $130 \mathrm{~km} / \mathrm{h}$ (highway configuration). The Extended Vehicular A (EVA) channel model is used for this simulation as it is suitable for cellular communications in a NLOS environment. Indeed, it describes propagation channel for studying LTE communications (see [17], [18]). Due to user mobility, the coherence time limits the available duration to acquire channel state information (CSI) and thereby limits the number of users too. Thus, we set the number of users to 5 (resp. 2) for $50 \mathrm{~km} / \mathrm{h}$ (resp. $130 \mathrm{~km} / \mathrm{h}$ ) in order to have less than $10 \%$ of the coherence time dedicated to the channel estimation. Under these conditions, the values of $R_{\infty}$ and $\tilde{R}_{\infty}$, defined in (28), are estimated through simulations. While $R_{\infty}=21.1 \mathrm{~b} / \mathrm{s} / \mathrm{Hz}$ for $50 \mathrm{~km} / \mathrm{h}$ and $R_{\infty}=17.3 \mathrm{~b} / \mathrm{s} / \mathrm{Hz}$ for $130 \mathrm{~km} / \mathrm{h}, \tilde{R}_{\infty}=10.7 \mathrm{~b} / \mathrm{s} / \mathrm{Hz}$ for both speed values. Therefore, $R_{\infty}>\tilde{R}_{\infty}$ and in this context the frequency

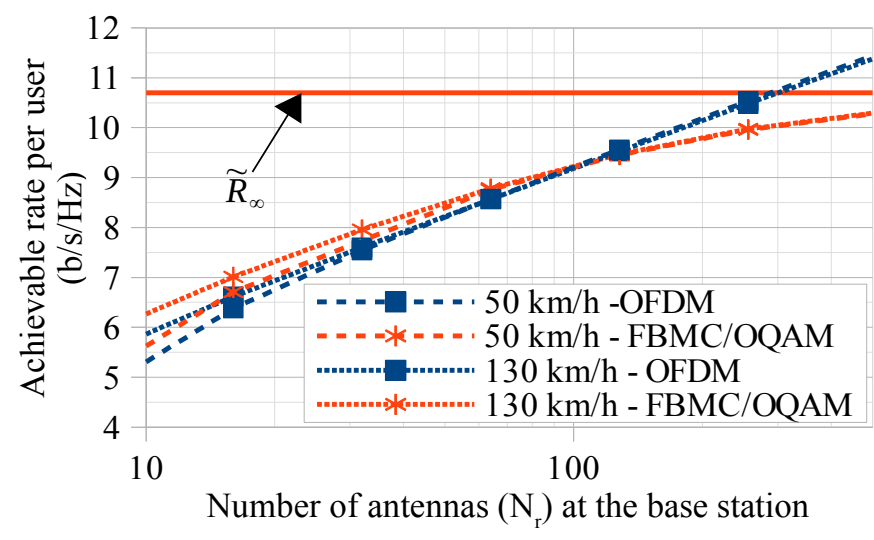

Fig. 4. Achievable rate per user as a function of the number of receive antennas $\left(N_{r}\right)$ with 5 users at $50 \mathrm{~km} / \mathrm{h}$ and 2 users at $130 \mathrm{~km} / \mathrm{h}$ and with the EVA channel model.

selectivity is predominant compared to the time selectivity of the channel and should limit the performance of the FBMC/OQAM modulation with high values of $N_{r}$. Figure 4 shows the achievable rate per user for the OFDM and the FBMC/OQAM modulations as a function of $N_{r}$.

Even with a speed of $130 \mathrm{~km} / \mathrm{h}$, we have $R \ll R_{\infty}$ and the Doppler effect has impact neither on the OFDM performance nor on the FBMC/OQAM one. With the FBMC/OQAM modulation, we can consider that the equations in (25) are valid for $N_{r} \leq 20$. This leads to a maximum gain of approximately $0.4 \mathrm{~b} / \mathrm{s} / \mathrm{Hz}$ with speed values of $50 \mathrm{~km} / \mathrm{h}$ and of $130 \mathrm{~km} / \mathrm{h}$ compared to the OFDM modulation. For higher values of $N_{r}$, the equations in (25) are not valid anymore and the FBMC/OQAM modulation is limited by its intrinsic interference. The difference $(\tilde{R}-R)$ thus decreases as $N_{r}$ increases and even becomes negative when $N_{r} \geq 100$. Regarding the central frequency, a base station with more than 100 uncorrelated antennas is actually very optimistic. Therefore, the FBMC/OQAM modulation performance is slightly above the OFDM performance for this scenario but other metrics such as complexity or spectral confinement should be considered.

\section{B. High speed train scenario}

Then, we study very high speed communications such as train connection with speed values from $150 \mathrm{~km} / \mathrm{h}$ to the limit defined by [2], $500 \mathrm{~km} / \mathrm{h}$. We choose a line-of-sight (LOS) channel model which better meets this type of high-speed scenario. The considered channel model called Rural Area is made up with 4 taps (RA4) and has a Rician K factor of 6.7 as defined in [19]. With such a mobility, the available time to estimate the propagation channel is very small and only one user can be considered. Moreover, the equations in (25) are not valid. In Figure 5, the achievable rate is computed as a function of the speed for the OFDM and FBMC/OQAM modulations and for $N_{r}=64, N_{r}=128$ and $N_{r}=\infty$.

With this LOS propagation channel and a speed above $150 \mathrm{~km} / \mathrm{h}$, one can see that $\tilde{R}_{\infty}>R_{\infty}$. Therefore the FBMC/OQAM modulation always outperforms the OFDM 


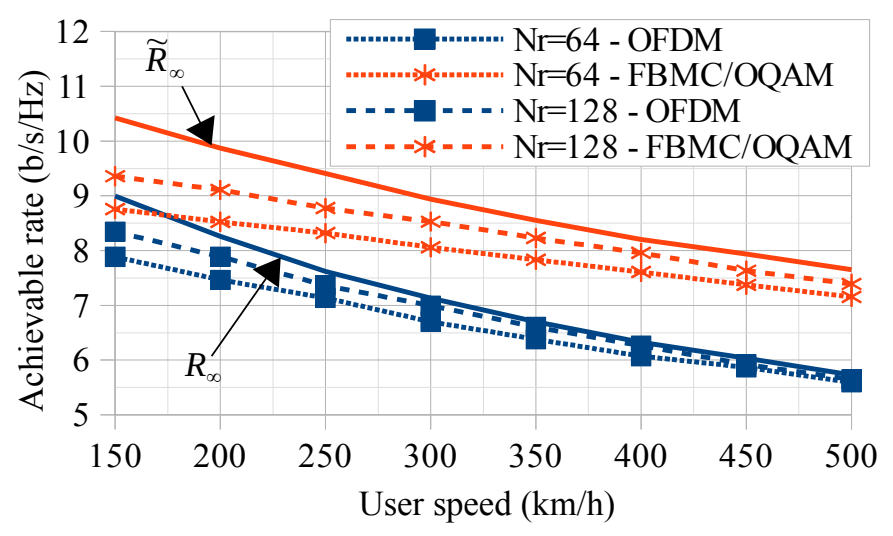

Fig. 5. Achievable rate as a function of the speed with 64 and 128 receive antennas at the base station side and with the RA4 channel model.

modulation whatever the value of the speed, of $\rho$ and of $N_{r}$. Moreover, the difference $(\tilde{R}-R)$ increases with the speed and, when the number of receive antennas tends towards infinity, the maximum value of $1.9 \mathrm{~b} / \mathrm{s} / \mathrm{Hz}$ can be reach. The FBMC/OQAM modulation is thus interesting in this high speed context. For example, with 64 receive antennas and a speed of $250 \mathrm{~km} / \mathrm{h}$, the FBMC/OQAM modulation can send $1.2 \mathrm{~b} / \mathrm{s} / \mathrm{Hz}$ more than the OFDM modulation.

\section{Conclusion}

This paper presents the impact of the Doppler effect on the capacity of an uplink communication associated with multiuser massive MIMO techniques at the base station side. The OFDM and the FBMC/OQAM modulations are considered.

With a moderate speed, we demonstrate that the lack of $\mathrm{CP}$ for the FBMC/OQAM modulation can be an advantage when the number of antennas at the base station is low. However, when this number is higher, the intrinsic interference created by the frequency selectivity of the channel becomes non-negligible and limits the performance of the FBMC/OQAM modulation. Simulation results show that the impact of the Doppler effect is actually not significant even at $130 \mathrm{~km} / \mathrm{h}$ and compared to the OFDM modulation performance, the FBMC/OQAM modulation performance is equivalent or slightly better in terms of achievable rate per user. Thus, the choice between these two modulations has to be done taking into account other metrics such as complexity or spectral confinement.

In very high mobility conditions, with a speed from 150 $\mathrm{km} / \mathrm{h}$ to $500 \mathrm{~km} / \mathrm{h}$, we demonstrate that the Doppler effect limits both the OFDM and the FBMC/OQAM modulations. However, thanks to its prototype filter (TFL1), the FBMC/OQAM modulation is actually less impacted as confirmed by the simulation results. Indeed, in terms of achievable rate, the OFDM modulation performance is always outperformed by the FBMC/OQAM modulation performance in this context. Therefore, the FBMC/OQAM modulation can significantly improve the capacity of a massive MIMO uplink system in very high mobility conditions.
This paper actually paves the way for deeper studies. The system model could be closer from a real system taking into account channel coding and synchronization processes and a real channel estimation for example. Similarly, a more general scenario can be considered with multiple-antenna users having heterogeneous speeds and variable distances to the base station.

\section{REFERENCES}

[1] “5g white paper," NGMN Alliance, Tech. Rep., Feb. 2015. [Online]. Available: ngmn.org

[2] "IMT Vision Framework and overall objectives of the future development of IMT for 2020 and beyond," ITU-R, Tech. Rep. M.2083-0, Sep. 2015. [Online]. Available: www.itu.int

[3] B. Hirosaki, "An Orthogonally Multiplexed QAM System Using the Discrete Fourier Transform," IEEE Transactions on Communications, vol. 29, no. 7, pp. 982-989, Jul. 1981.

[4] P. Siohan, C. Siclet, and N. Lacaille, "Analysis and design of OFDM/OQAM systems based on filterbank theory," IEEE Transactions on Signal Processing, vol. 50, no. 5, pp. 1170-1183, May 2002.

[5] T. Fusco, A. Petrella, and M. Tanda, "Sensitivity of multi-user filterbank multicarrier systems to synchronization errors," in 3rd International Symposium on Communications, Control and Signal Processing, 2008. ISCCSP 2008, Mar. 2008, pp. 393-398.

[6] R. Haas and J.-C. Belfiore, "A Time-Frequency Well-localized Pulse for Multiple Carrier Transmission," Wireless Personal Communications, vol. 5, no. 1, pp. 1-18, Jul. 1997.

[7] T. Strohmer and S. Beaver, "Optimal OFDM design for time-frequency dispersive channels," IEEE Transactions on Communications, vol. 51, no. 7, pp. 1111-1122, Jul. 2003.

[8] B. Farhang-Boroujeny, "OFDM Versus Filter Bank Multicarrier," IEEE Signal Processing Magazine, vol. 28, no. 3, pp. 92-112, May 2011.

[9] E. Larsson, O. Edfors, F. Tufvesson, and T. Marzetta, "Massive MIMO for next generation wireless systems," IEEE Communications Magazine, vol. 52, no. 2, pp. 186-195, Feb. 2014.

[10] H. Q. Ngo, E. Larsson, and T. Marzetta, "Energy and Spectral Efficiency of Very Large Multiuser MIMO Systems," IEEE Transactions on Communications, vol. 61, no. 4, pp. 1436-1449, Apr. 2013.

[11] T. Marzetta, "Noncooperative Cellular Wireless with Unlimited Numbers of Base Station Antennas," IEEE Transactions on Wireless Communications, vol. 9, no. 11, pp. 3590-3600, Nov. 2010.

[12] H. Q. Ngo, T. L. Marzetta, and E. G. Larsson, "Analysis of the pilot contamination effect in very large multicell multiuser MIMO systems for physical channel models," in 2011 IEEE International Conference on Acoustics, Speech and Signal Processing (ICASSP), May 2011, pp. 3464-3467.

[13] J. Hoydis, S. ten Brink, and M. Debbah, "Massive MIMO in the UL/DL of Cellular Networks: How Many Antennas Do We Need?" IEEE Journal on Selected Areas in Communications, vol. 31, no. 2, pp. 160-171, Feb. 2013.

[14] A. Farhang, N. Marchetti, L. Doyle, and B. Farhang-Boroujeny, "Filter Bank Multicarrier for Massive MIMO," in Vehicular Technology Conference (VTC Fall), 2014 IEEE 80th, Sep. 2014, pp. 1-7.

[15] A. Aminjavaheri, A. Farhang, N. Marchetti, L. Doyle, and B. FarhangBoroujeny, "Frequency spreading equalization in multicarrier massive MIMO," in 2015 IEEE International Conference on Communication Workshop (ICCW), Jun. 2015, pp. 1292-1297.

[16] D. Pinchon and P. Siohan, "Derivation of analytical expressions for flexible PR low complexity FBMC systems," in Signal Processing Conference (EUSIPCO), 2013 Proceedings of the 21st European, Sep. 2013, pp. 1-5.

[17] "User Equipment (UE) Radio Transmission and Reception." 3GPP, Tech. Rep. TS 36.101. [Online]. Available: www.3gpp.org

[18] "Base Station (BS) Radio Transmission and Reception." 3GPP, Tech. Rep. TS 36.104. [Online]. Available: www.3gpp.org

[19] "Digital land mobile radio communications," COST, Tech. Rep. COST 207, 1989. 